\title{
Self-Referencing Fiber-Optic Intensity Sensors Using Ring Resonators and Fiber Bragg Gratings
}

\author{
C. Vázquez, Senior Member, IEEE, J. Montalvo, D. S. Montero, and J. M. S. Pena, Senior Member, IEEE
}

\begin{abstract}
An improved ring resonator self-referencing technique in a new reflection configuration for remote fiber-optic intensity sensors is demonstrated using fiber Bragg gratings. Sensor sensitivity doubles and a single fiber lead is used. The sensor is interrogated at two subcarrier frequencies having a high rejection of interference from laser source intensity fluctuations and loss in the fiber lead. We experimentally demonstrate the efficiency of the new reflection configuration, the usefulness of the theoretical model proposed, and discuss design parameters for optimum insertion losses.
\end{abstract}

Index Terms-Fiber-optic sensor, intensity-based sensor, microfibers, ring resonator, self-referencing.

\section{INTRODUCTION}

$\mathbf{I}$ NTRINSICALLY safe sensors based on multimode (MM) and single-mode (SM) fibers can be made for a wide variety of physical magnitudes. Simple fiber-optic intensity sensors (FOS) modulate the loss of a fiber path to provide the measurement as an optical intensity modulation signal. A serious drawback of loss modulation is interference from variation in loss due to long-term aging of optical source as long as short-term fluctuations of optical power loss in the fiber lead which can occur, for example, in fiber bends. A reference signal is needed for calibration out of the sensor response, which undergoes all other losses in the system, and which is multiplexed in the same fiber lead as the measurement signal. Time division, wavelength normalization [1], [2], and frequency-based self-referencing methods [3], [4], all based on MM fibers, have been reported to overcome this problem. More recently, a method using counterpropagating signals [5], Michelson [6], and ring resonator (RR) [7] topologies have been proposed with SM fibers. In the RR topology with SM fibers [7], an optical RR with an FOS in the RR cavity is placed at each remote measuring point and two fiber leads are used.

In this letter, an RR self-referencing technique in a new reflection configuration is proposed. The configuration has the doubled sensitivity in comparison with an RR transmission configuration [7], uses a single fiber lead for interrogating the sensor, can be easily adjusted in comparison with two RR in cascade, and opens up wavelength-division-multiplexing capabilities. A circulator is used to extract the reflected signal coming back to the central office in the same fiber lead, reducing one of the noise sources and simplifying the schematic. A theoretical model describing the configuration and the measurement parameter of the

Manuscript received April 11, 2006; revised September 5, 2006. This work was supported in part by CICYT (TIC2003-03783 and TEC2006-13273-C03-03-MIC), in part by UC3M (FAVICOBIS), in part by CAM (FACTOTEM-CM:S-0505/ESP/000417), and in part by COST 299.

The authors are with the Electronics Technology Department, Carlos III University of Madrid, 28911 Leganés, Spain (e-mail: cvazquez@ing.uc3m.es).

Digital Object Identifier 10.1109/LPT.2006.885640

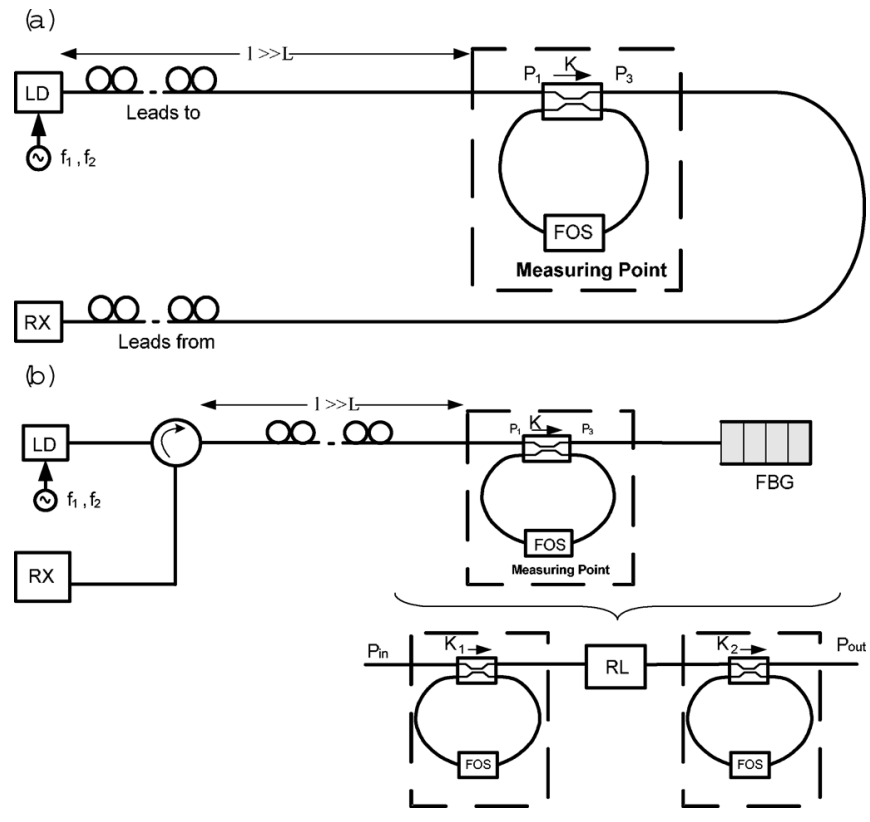

Fig. 1. RR self-referencing technique schematic for remote operation. (a) Transmission configuration. (b) Reflection configuration.

self-referencing technique is reported. A discussion of design parameters depending on total insertion losses is included. And experimental measurements are taken to validate the equations, and the rejection to fiber lead attenuation of the new self-referencing technique configuration. The technique can be used in microfiber loop resonators [8], where the microfiber loop is the sensing element itself.

\section{THEORY}

The RR self-referencing technique in a transmission configuration [3], [7] is made of an RR with an optical fiber sensor, FOS, in the loop and it is placed at each remote measuring point [see Fig. 1(a)]. By adding a fiber Bragg grating (FBG) at the $\mathrm{RR}$ output fiber or microfiber loop, a reflection configuration is made [see Fig. 1(b)]. In both cases, $K$ is the direct power coupling factor of the optical coupler, $L$ is the loop length, and the global loop power balance $H$ is given by

$$
H=10^{-\alpha \cdot L / 10} \cdot F(m) \cdot(1-\gamma)
$$

where $\gamma$ represents the coupler excess losses; $\alpha$ is the power losses of optical fiber in decibels per meter; and $F(m)$ is the optical fiber sensor calibration versus the external magnitude $m$.

The novel configuration can be analyzed in coherent or incoherent regimen; in the following, we are going to consider that the optical source coherence length is much lower than 
loop length $L$, so incoherent optical interference regime is performed. The symmetric decomposition shown in Fig. 1(b), in two RR in series, assuming bidirectional intensity sensor response, can be used to study the structure, where $P_{\text {in }}=P_{1}^{+}$ is the forward input optical power at Port 1 and $P_{\text {out }}=P_{1}^{-}$the backward output optical power at Port 1. $R L$ represents FBG reflection losses. This configuration optimizes the second-order filter response in terms of symmetry, because the two rings are physically the same, and the same point of operation for the two equivalent resonators can be achieved with one sole adjusting process as regards coupling factor $\left(K_{1}=K_{2}\right)$ and loop length $\left(L_{1}=L_{2}\right)$. Because of that, the configuration is insensitive to coupling factor and loop length fabrication tolerances for symmetric bidirectional optical couplers and intensity sensors. The $Z$-transform magnitude of the power transfer function impulse response is given by

$$
\left|H_{p}(z)\right| \equiv\left|\frac{P_{\text {out }}}{P_{\text {in }}}\right|=\Gamma\left|\frac{z-z_{c}}{z-z_{p}}\right|^{2}
$$

where

$$
\begin{aligned}
\Gamma & =R L \cdot K^{2} \cdot(1-\gamma)^{2} \\
z_{c} & =\frac{(2 \cdot K-1) H}{K} \\
z_{p} & =K \cdot H .
\end{aligned}
$$

Assuming that input optical power is constant as a function of normalized pulsation $\Omega=2 \cdot \pi \cdot f \cdot \tau$, where $f$ is the modulation radio frequency (RF) and $\tau$ the loop transit time, the self-referencing parameter $R_{M, n}$, defined as in [7] for the new configuration and for a generic node $n$, is given by

$$
R_{M, n}(\Omega) \equiv \frac{\left|\frac{P_{\mathrm{out}, n}}{P_{\mathrm{in}, n}}\right|_{z=\exp (j \Omega)}}{\left|\frac{P_{\mathrm{out}, n}}{P_{\mathrm{in}, n}}\right|_{\substack{z=\exp (j \Omega) \\ \Omega=2 \cdot \pi \cdot f_{2} \cdot \tau}}}=\left|\frac{P_{\mathrm{out}, n}(\Omega)}{P_{\mathrm{out}, n\left(\Omega=2 \cdot \pi \cdot f_{2} \cdot \tau\right)}}\right| .
$$

A differential measurement is achieved by modulating optical channels with two RFs $f, f_{2}$ and using an RR in a reflection configuration at each measuring point; where $f_{2}$ is the reference signal for calibration, and $f$ the sensor signal depending on sensor response $F(m)$.

From (2) and (4), it is derived that the reference frequency is given by

$$
f_{2}=\frac{i}{\tau}, \quad i=0,1,2 \ldots
$$

and the $H$ value for the resonance condition, making $/ H_{p}(z)=$ $0 /$, and named $H_{\text {zero }}$ is given by

$$
H_{0}=\frac{K}{1-2 \cdot K} .
$$

So $f_{2}$ and $H_{o}$ are the same ones as in the RR transmission configuration described in [7].

RR transmission and reflection configuration normalized frequency responses can be seen at Fig. 2; reference $\left(f_{2}\right)$ and sensor $(f)$ frequencies are marked. There is a ratio of two between theoretical responses of RR reflection and transmission configuration, as expected from (2).

It is important to determine the design restrictions of the technique. It is derived from (8) that the resonance condition $H_{\text {zero }}$ is achieved without using gain only for $K<1 / 3$. On the other

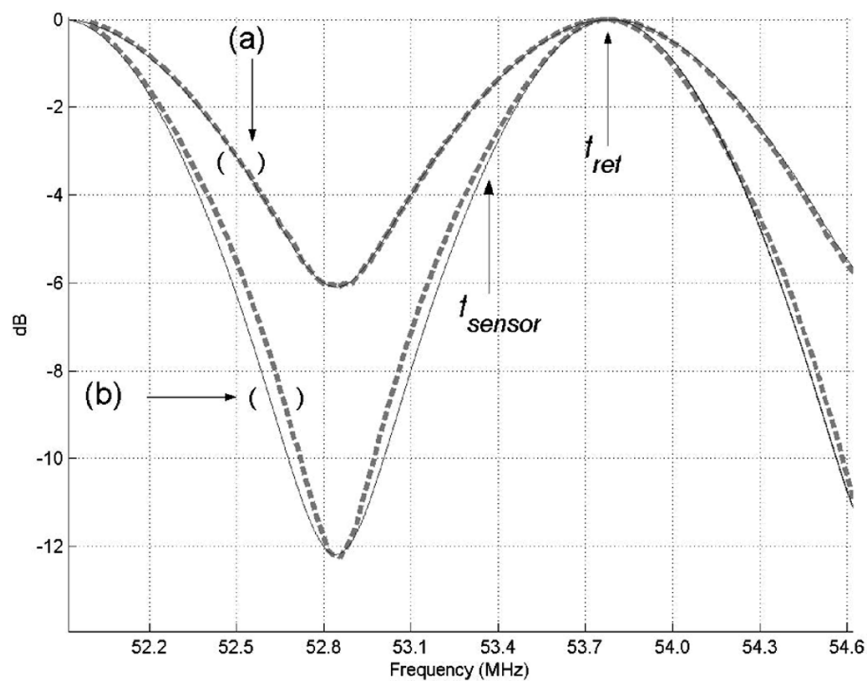

Fig. 2. Theory (-) and measurements (- - ) of normalized RR RF magnitude response with $K=0.207, H=0.7$. (a) Transmission configuration. (b) Reflection configuration.

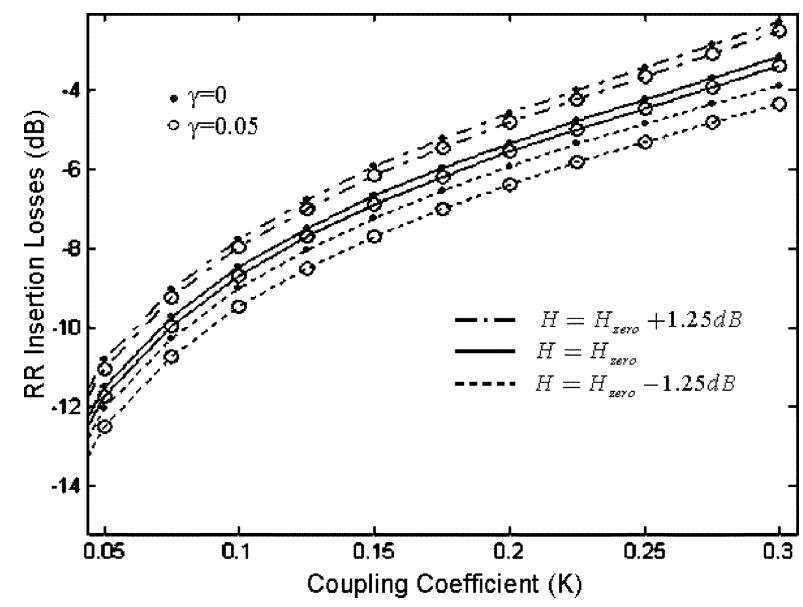

Fig. 3. RR insertion losses, $\mathrm{IL}_{\mathrm{RR}}$, as a function of $K$ in the condition of minima $\left(H=H_{\text {zero }}\right)$ and for deviations of $1.25 \mathrm{~dB}\left(H=H_{\text {zero }} \pm \Delta\right)$ and $\gamma=0,0.05$.

hand, $R R$ insertion losses $\mathrm{IL}_{\mathrm{RR}}$ can be derived using Parseval's identity

$\mathrm{IL}_{\mathrm{RR}}=\frac{1}{N} \cdot \sum_{i=0}^{N-1}\left|H\left(z=\exp \left(\frac{j 2 \cdot \pi \cdot i}{N}\right)\right)\right|^{2}, \quad N \rightarrow \infty$.

They have been plotted versus $K$ in the resonance condition $H=H_{\text {zero }}$ and for deviations $\Delta$ of $1.25 \mathrm{~dB}\left(H=H_{\text {zero }} \pm \Delta\right)$ using $\gamma$ as the parameter (see Fig. 3). Those insertion losses double in the RR reflection configuration.

\section{MEASUREMENTS}

The RR self-referencing technique in transmission and reflection configuration shown in Fig. 1 is implemented with standard SM fiber with angled physical contact fiber connector ( $\mathrm{FC} /$ APC) connectors, and FBG from O/E Land INC (using index matching gel at its transmission port). FOS is simulated by a variable optical attenuator (VOA). RR loop length is $100 \mathrm{~m}$ (without considering coupler and VOA pigtails) to ensure incoherent operation, a fixed optical coupler is used to ensure 


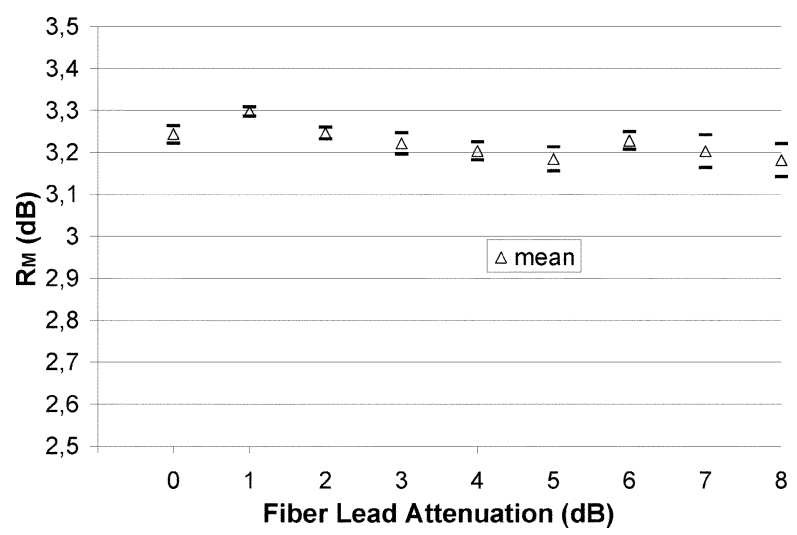

Fig. 4. $R_{M}$ measurements versus fiber lead induced attenuation for an RR selfreferencing technique in reflection configuration with $K=0.207, \gamma=0.05$, $H=0.7 . f_{2}=53.86 \mathrm{MHz}$ (reference), $f=53.35 \mathrm{MHz}$ (sensor).

bidirectional operation in comparison with more unstable variable ratio couplers. A laser diode (LD) at $1552.2 \mathrm{~nm}$ is used as the optical carrier. This LD is externally modulated with an RF signal generated inside the 8703B Lightwave Component Analyzer (LCA) which is also used for measuring simultaneously the configuration response at different RF frequencies. LCA can operate in optical/optical mode for characterizing the optical device under test from $50 \mathrm{MHz}$ to $20 \mathrm{GHz}$. A frequency window of $4.5 \mathrm{MHz}$ with 201 points and $100-\mathrm{Hz}$ intermediate frequency filter is used in the measurements. A circulator is used for extracting the backward signal in the lead fiber. Two sets of measurements are taken, first to validate the theoretical model and second to verify the isolation of the measurements from the effects of fiber lead attenuation and LD power fluctuations in the reflection configuration. $K$ values are selected for avoiding using optical amplification and having acceptable RR insertion losses.

An RR configuration with $K=0.207, H=0.7, \gamma=0.05$, has been characterized (see Fig. 2). A good agreement can be seen between theory and measurements. freqz MATLAB function is used in simulations. In the reflection configuration, there is a small deviation related to coupler and VOA asymmetries for the backward and forward propagation. RR transmission configuration is measured interchanging input and output port $\left(P_{1}\right.$ and $P_{3}$ in Fig. 1). In these measurements, the shape of the response is kept and there is just a $0.1-\mathrm{dB}$ variation in the amplitude of the minima. The double sensitivity for the reflection configuration is also verified.

On the other hand, RR insertion losses are measured with an optical power meter for two different $K$ and $H$ (close to $H_{\text {zero }}$ ) values in each case; results are in accordance with simulations (at $K=0.07$, it is measured $\mathrm{IL}_{\mathrm{RR}}=11 \mathrm{~dB}$; at $K=0.19$, it is measured $\mathrm{IL}_{\mathrm{RR}}=7.7 \mathrm{~dB}$ ) (see Fig. 3)

On validating the self-referencing technique, we emulated the main fiber lead power fluctuations by using another VOA in the fiber lead connecting the optical source to the self-referencing sensor, adjusting the attenuation to induce losses from 0 to $8 \mathrm{~dB}$. A set of $R_{M}$ measurements are taken for each fiber lead attenuation value for an RR reflection configuration with $K=0.207$, $f=53.35 \mathrm{MHz}$, and $f_{2}=53.86 \mathrm{MHz}$. Mean, maxima, and minima $R_{M}$ values are reported in Fig. 4, having a relative error below $0.6 \%$.
System resolution in terms of optical loss in $\mathrm{dB} / \sqrt{ } \mathrm{Hz}$ is around $0.002 \mathrm{~dB} / \sqrt{ } \mathrm{Hz}$ in the same order of magnitude of the values reported in [9]. The sensing concept is particularly favorable in what concerns the minimization of system noise, because what is monitored is the amplitude of two sinewaves, i.e., the detection bandwidth can be made as narrow as practically possible, with consequent reduction of the system noise level.

An important aspect is keeping FC/APC connectors properly aligned to avoid undesired reflections; final prototypes will avoid any connector by using splices. Sensor frequency values closer to the resonance frequency have higher relative errors. In future measurements, polarization-maintaining fiber and couplers will be used to delimit the influence of loop fiber birefringence on measurements.

\section{CONCLUSION}

An RR self-referencing technique in a new reflection configuration for remote FOS is demonstrated using FBGs. The configuration has the doubled sensitivity in comparison with a transmission configuration, uses a single fiber lead for interrogating the sensor, can be easily adjusted in comparison with two RR in cascade. Experimental normalized frequency responses of transmission and reflection configurations are reported validating the theory. The sensor is interrogated at two RF frequencies (reference and sensor) having a high rejection of interference from optical power fluctuations. The reflection configuration has been tested by an induced noise of up to $8-\mathrm{dB}$ power fluctuation in the fiber lead at different sensor frequencies. Relative errors lower than $0.6 \%$ in the measurement parameter are reported. Equations validated with measurements are reported to optimize RR reflective configuration insertion losses, where this parameter increased in the new configuration. Multiplexing capabilities are opened by using FBG in a bus or star topology in reflective configurations [6]-[10].

\section{REFERENCES}

[1] J. W. Berthold, III, "Historical review of microbend fiber-optic sensors," J. Lightw. Technol., vol. 13, no. 7, pp. 1193-1199, Jul. 1995.

[2] W. B. Spillman and J. R. Lord, "Self-referencing multiplexing technique for fiber-optic intensity sensors," J. Lightw. Technol., vol. LT-5, no. 7, pp. 865-869, Jul. 1987.

[3] R. I. MacDonald and R. Nychka, "Differential measurement technique for fiber-optic sensors," Electron. Lett., vol. 27, pp. 2194-2196, 1991.

[4] J. M. Baptista, J. L. Santos, and A. S. Lage, "Mach-Zehnder and Michelson topologies for self- referencing fiber optic intensity sensors," Opt. Eng., vol. 39, pp. 1636-1644, 2000.

[5] C. S. Pérez, A. G. Valenzuela, G. E. S. Romero, J. Villatoro, and J. H. Cordero, "Technique for referencing of fiber-optic intensity modulated sensors by use of counterpropagating signals," Opt. Lett., vol. 29, pp. 1467-1469, 2004.

[6] J. M. Baptista, S. Abad, G. M. Rego, L. A. Ferreira, F. M. Araujo, and J. L. Santos, "Wavelength multiplexing of frequency-based self-referenced fiber optic intensity sensors," Opt. Eng., vol. 43, pp. 702-707, 2004.

[7] C. Vázquez, J. Montalvo, and P. C. Lallana, "Radio-frequency ring resonators for self-referencing fibre-optic intensity sensors," Opt. Eng. Lett., vol. 44, pp. 1-2, 2005.

[8] M. Sumetsky, Y. Dulashko, J. M. Fini, A. Hale, and D. J. DiGiovanni, "The microfiber loop resonator: Theory, experiment, and application," J. Lightw. Technol., vol. 24, no. 1, pp. 242-250, Jan. 2006.

[9] J. M. Baptista, J. L. Santos, and A. S. Lage, "Measurement of refractive index in oils using self-referenced fiber optic intensity sensor," in LEOS 2001, 2001, vol. 2, pp. 875-876.

[10] J. Montalvo, C. Vázquez, and D. S. Montero, "CWDM self-referencing sensor network based on ring resonators in reflective configuration," Opt. Express, vol. 14, pp. 4601-4610, 2006. 